\title{
A kinematical catalogue of HII regions and superbubbles in the LMC
}

\author{
P. Ambrocio-Cruz ${ }^{1}$, E. Le Coarer ${ }^{2}$, M. Rosado ${ }^{3}$, D. Russeil ${ }^{4}$, \\ P. Amram ${ }^{4}$, A. Laval $^{4}$, B. Epinat ${ }^{4}$, \\ M. Ramírez ${ }^{1}$, M. Odonne ${ }^{5}$ and G. Goldes ${ }^{5}$ \\ ${ }^{1}$ AA Ciencias de la Tierra y Materiales Universidad Autónoma del Estado de Hidalgo, México \\ email: patricia@astro.unam.mx \\ ${ }^{2}$ Laboratoire d'Astrophysique de Grenoble, France \\ ${ }^{3}$ Instituto de Astronomía, UNAM, México \\ email: margarit@astro.unam.mx \\ ${ }^{4}$ Laboratoire d'Astrophysique de Marseille, France \\ ${ }^{5}$ Observatorio Astronómico Laprida, Argentina
}

\begin{abstract}
We report the results of a kinematic $\mathrm{H} \alpha$ survey of the Large Magellanic Cloud (LMC) in the form of a kinematic and photometric catalogue of 210 HII regions, the radial velocity field of the ionized hydrogen in this galaxy, and the LMC Rotation Curve obtained from the velocity field. These data aim at understanding the LMC HII regions, bubbles and superbubbles in a global (galactic) scale so that we could have a $3 \mathrm{D}$ view and separate the rotation due to gravitational potential from other motions such as expansions.
\end{abstract}

Keywords. galaxies: Magellanic Clouds, ISM: kinematics and dynamics, ISM: bubbles, supernova remnants

We have carried out a kinematic $\mathrm{H} \alpha$ survey of the LMC. The observations were made with a scanning Fabry-Perot interferometer which produced 3D data cubes for 69 pointings over the LMC, each with a FOV of 38 arcmin. The data cubes were flux calibrated through HII regions with known $\mathrm{H} \alpha$ intensity. Wavelength calibration and astrometry have also been carried out. Each data cube provides radial velocity fields of ionized gas in the LMC and in the Galaxy.

We find a bimodal distribution of the $\mathrm{H} \alpha$ luminosity of LMC HII regions, possibly due to a transition between radiation-bounded and density-bounded HII regions. From the analysis of this large sample of HII regions, we suggest that triggered star formation in the LMC could amount to $48 \%$ and that this mechanism seems to be more active in small (young) and density-bounded HII regions. We also derive the local star formation rate of the LMC that agrees quite well with those derived from other tracers.

There is a very good agreement between the rotation curve (RC) we derived from the ionized gas and the RCs derived from other tracers (stars, planetary nebulae, neutral gas), except that for $\mathrm{R}>2.5 \mathrm{kpc}$, HI kinematics starts to deviate towards lesser rotation velocities. Most of the catalogued nebulae follow the RC well, except 30 Dor, N119, LMC 4 and N 44D. These deviations from circular motions may have different causes, such as out of disk location (as it has been proposed for the 30 Dor Nebula), the presence of a prominent bar and inflowing gas, and expansion motions due to supernova explosions. We find no correlation among the velocity dispersion, the size, and the $\mathrm{H} \alpha$ intensity of the HII region. Finally, we note that nebulae with large velocity dispersions correspond to known supernova remnants. 